\title{
EDITORIAL
}

\section{Un año de incertidumbres para la Educación Matemática}

\author{
Vicenç Font * \\ ORCID iD 0000-0003-1405-0458 \\ Gemma Sala** \\ ORCID iD 0000-0001-9830-312X
}

Aunque durante este año se ha hablado, reflexionado e investigado ampliamente sobre el efecto de la pandemia de la COVID19 en la Educación Matemática, en cierta manera es inevitable, en esta editorial del último número de Bolema del año 2020, continuar hablando de esta problemática, dado que parece que la pandemia nos va a acompañar más tiempo del que, en algún momento, se pensó. Al escribir esta editorial ya parece claro que el 2021 será un año lleno de incertidumbres como consecuencia de la pandemia, en particular lo será para la Educación Matemática.

En la editorial del número anterior de Bolema (escrito durante lo que se llamó, seguramente de manera no muy precisa, la primera oleada de la pandemia) Miarka y Maltempi (2020) se preguntaban ¿qué será de la Educación Matemática después del coronavirus? En estos momentos parece que la respuesta es que la investigación en Educación Matemática será diferente en muchos aspectos que ya, más o menos, sabemos cuáles son y que lo será, también, en otros que aún no conocemos.

Un primer aspecto, previsiblemente, será un aumento de la investigación sobre las acciones que tomaron los profesores en general (y los de matemáticas en particular) en este periodo de pandemia. Al inicio de la pandemia, una de las medidas adoptadas para afrontarla

\footnotetext{
* Dr. en Filosofía y Ciencias de la Educación por la Universitat de Barcelona (UB). Profesor titular de universidad del Departamento de Educación Lingüística y Literaria y de Didáctica de las Ciencias Experimentales y de la Matemática de la Facultad de Educación de la Universitat de Barcelona (UB), Barcelona, Catalunya, España. Dirección postal: Campus Mundet Passatge de la Vall d’Hebrón, nº 171, 08035, Barcelona, España. E-mail: vfont@ub.edu.

${ }^{* *}$ Dra. en Didáctica de las Matemáticas por la Universitat de Barcelona (UB). Profesora asociada al Departamento de Educación Linguística y Literaria y de Didáctica de las Ciencias Experimentales y de la Matemática de la Facultad de Educación de la Universitat de Barcelona (UB), Barcelona, Catalunya, España. Dirección postal: Campus Mundet Passatge de la Vall d'Hebrón, nº 171, 08035, Barcelona, España. E-mail: gsala @ub.edu.
} 
fue el confinamiento de la población en sus casas y el cierre de los centros educativos. En los diferentes países se siguió una trayectoria similar y se optó por el uso de las tecnologías para continuar con la docencia, de manera virtual (ZHOU et al., 2020). Esta situación fue un reto para los docentes, que tuvieron que proseguir con el temario, afrontando el desafío de la distancia, la posibilidad de conexión de sus estudiantes (o de ellos mismos/as), las situaciones personales, familiares, el uso de unas herramientas para las que se tuvieron que formar, tanto ellos como sus estudiantes, sobre la marcha etc. Toda esta situación ha tenido un profundo impacto sobre el aprendizaje y la enseñanza, del que todavía no conocemos el alcance y sobre el que, en estos momentos, se están realizando investigaciones con muchos enfoques diferentes (DIBNER; SCHWEINGRUBER; CHRISTAKIS, 2020; ZHANG et al., 2020; ZHOU et al., 2020).

Si bien hay debate sobre la efectividad del cierre de las escuelas como medida para controlar la pandemia (de hecho, en la llamada segunda ola de la pandemia se procura mantener la escuela abierta todo lo posible), en lo que sí parece haber consenso es que las medidas tomadas tienen mayor impacto en los grupos de población más vulnerables (VAN LANCKER; PAROLIN, 2020). Por esta razón, es importante conocer, con precisión, qué efecto ha tenido, primero, el cierre de las escuelas y, después, el paso a una enseñanza de tipo híbrido. Pero también es importante identificar actuaciones de éxito que se han dado en esta situación (por ejemplo, los profesores han diseñado tareas para explicar la evolución de la actual pandemia mediante modelos matemáticos), porque no sabemos si en los próximos meses vamos a vivir situaciones similares de confinamiento o bien situaciones de enseñanza híbrida. Mientras que no existan tratamientos efectivos contra el virus de la COVID-19 o una vacuna efectiva con acceso universal, es preciso aprender de las lecciones vividas, para poder extraer aprendizajes útiles, contrastados por un proceso de investigación riguroso, y que puedan orientar, de manera idónea (BREDA; FONT; PINO-FAN, 2018), la práctica del profesor de matemáticas en el próximo año y en situaciones futuras similares.

En el ámbito de la enseñanza y aprendizaje de las matemáticas tenemos bastante conocimiento sobre el uso de las tecnologías como recurso educativo (JOSHI, 2017; LABORDE， 2007; VERSCHAFFEL; DEPAEPE; MEVARECH，2019). En estos trabajos, la tecnología aparece como herramienta. Pero la situación actual plantea un nuevo escenario, donde la tecnología no es solo la herramienta, sino que se convierte en el medio a través del cual se establece la relación entre docentes y estudiantes. Los trabajos sobre la tecnología como medio, en el ámbito de las matemáticas, son mucho más restringidos. Tenemos pocas evidencias sobre cómo afecta, por ejemplo, el uso de entornos virtuales al aprendizaje de 
las matemáticas escolares desde el punto de vista del aprendizaje y de los resultados de aprendizaje. Esto no quiere decir que no existan multitud de plataformas digitales para la enseñanza y aprendizaje de las matemáticas, y documentos que explican cómo funcionan técnicamente. Tenemos una gran multitud de plataformas digitales (comerciales y no comerciales), educadores on-line (algunos muy populares), recursos ofrecidos por las administraciones educativas, aplicaciones basadas en inteligencia artificial etc. Investigaciones sobre el uso de: tecnologías o plataformas de aprendizaje y enseñanza virtuales; entornos o modalidades de enseñanza y aprendizaje a distancia (blended learning, open distance learning etc.) (PRATES; MATOS, 2020); contextos de enseñanza y aprendizaje fuera de la escuela etc. ofrecen aportes relevantes en un contexto de pandemia como el actual, donde no sabemos si la docencia, en el futuro inmediato, continuará siendo presencial, híbrida o totalmente online.

Por lo tanto, un segundo aspecto que nos dejará la pandemia será un aumento de la investigación sobre la enseñanza y aprendizaje de las matemáticas cuando la tecnología, además de ser un recurso, es el medio. En la gran mayoría se trata de herramientas de uso que se centran en fomentar la idoneidad epistémica de la matemática, y a veces, también en la idoneidad cognitiva del aprendizaje. Pero en cambio, otras idoneidades (BREDA; FONT; PINO-FAN, 2018) del aprendizaje y la enseñanza de las matemáticas, como por ejemplo la interaccional, la afectiva o la ecológica (la relación con el entorno), que han estado ampliamente estudiadas en la enseñanza presencial, y que sabemos que tienen un impacto muy relevante sobre el aprendizaje, quedan en un segundo plano cuando el proceso de instrucción se hace mediante estas herramientas digitales.

Un tercer aspecto es que es probable que esta pandemia ponga de manifiesto la necesidad de investigar con más determinación: 1) problemáticas que no se han considerado previamente, o bien se han dejado de lado (CASTRO et al., en prensa), 2) áreas que ya están siendo muy investigadas, pero que la pandemia ha puesto especialmente el foco en ellas. Me refiero, por ejemplo, a que se pondrá el foco no solo en los recursos que pueden favorecer el proceso de enseñanza y aprendizaje de las matemáticas, sino también en el acceso a dichos recursos (en regiones remotas, en grupos vulnerables, en comunidades indígenas, en suburbios de grandes ciudades, en cómo ciertos recursos permiten la accesibilidad a un mayor número de estudiantes según las necesidades de sus contextos etc.); a la necesidad de plantear cambios curriculares teniendo en cuenta los recursos tecnológicos mediante los cuales se va a implementar; a la necesidad de formación del profesorado de matemáticas para adaptarse a situaciones de enseñanza virtual o híbrida; incrementar la investigación sobre matemáticas y valores, matemáticas y ciudadanía, los procesos de subjetivación relacionados con la 
instrucción matemática etc.; el papel que pueden jugar las familias en la educación matemáticas de sus hijos; cambios en las políticas educacionales de los países; aumento de la investigación sobre la modelización matemática en dos direcciones: la enseñanza de la competencia de modelización y el uso de la modelización para enseñar contenidos matemáticos; el papel de los aspectos emocionales y afectivos en una enseñanza virtual o híbrida etc.

Un cuarto aspecto sobre el que no está claro el impacto es en la producción de tesis de maestría, tesis de doctorado, proyectos I+D en marcha, publicación de artículos de investigación en revistas etc. Por ejemplo, Miarka y Maltempi (2020) comentan que, en los primeros meses de confinamiento, el número de manuscritos enviados a Bolema para su evaluación había aumentado, pero resaltan que se trata de informes de investigaciones desarrolladas en un período anterior a la pandemia, básicamente en situaciones de enseñanza presencial (es plausible suponer que, para algunos investigadores, esta situación de aislamiento debido a la pandemia mejora su productividad; algo parecido de lo que se comenta sobre Newton, quién hizo afirmaciones de que algunos de sus principales descubrimientos tuvieron lugar durante un período de confinamiento). Otro ejemplo, es que muchas revistas han realizado llamadas a la comunidad investigadora para que les envíen artículos para monográficos sobre la Educación Matemática en este periodo de pandemia. Ahora bien, hay otros efectos como son la interrupción de tesis de doctorado y proyectos de investigación I+D por no poder realizar el trabajo de campo previsto en modalidad presencial que afectaran negativamente a la producción de publicaciones. Si bien, es claro que la pandemia está afectando a la producción científica, no es muy claro, aún, cómo lo está haciendo.

Un quinto aspecto en el que ya se nota el impacto de la pandemia es en el tipo de congresos que hará la comunidad interesada en la Educación Matemática. Durante un cierto periodo de tiempo los congresos presenciales que movilizaban a muchos profesores e investigadores como el ICME o el PME a nivel mundial y el CIBEM, el CIAEM o la RELME a nivel latinoamericano no se podrán realizar presencialmente, o lo harán con una asistencia muy inferior a la que tenían antes de la pandemia, por diferentes razones: recomendaciones sanitarias, aumento del coste de los pasajes de avión, dificultad para conseguir los permisos de la institución educativa etc. Durante un tiempo indeterminado los congresos serán en modalidad virtual, lo cual llevará a la aparición descentralizada de muchos nuevos congresos (por áreas geográficas, por temáticas de investigación, por nivel educativo, por el uso de un determinado marco teórico etc.) y, también se dará la posibilidad de que congresos de tipo regional, que antes se hacían presencialmente, puedan pasar a ser congresos internacionales de primer nivel con conferenciantes de diferentes partes del mundo. Un buen ejemplo, entre otros, lo tenemos 
con el 5to Encuentro Internacional de Investigación en Educación Matemática- EIEM5, un congreso gratuito para los asistentes, organizado durante el mes de octubre del 2020 por la Universidad del Atlántico (Barranquilla, Colombia), donde los organizadores pudieron reunir conferenciantes de reconocido prestigio como Ubiratan D’Ambrosio, Ole Skovsmose, Marcelo Borba, Saddo Ag Almouloud, Miriam Godoy Penteado, Roger Miarka, Adriana Breda, Bruno D’Amore, Luis Carlos Arboleda, Edelmira Badillo, Leonor Camargo, Pedro Gómez, Solange Roa, Carolina Tamayo, Jhony A. Villa-Ochoa, Claudia Vázquez, Marcel David Pochulu, Michele Artigue, Carmen Batanero, Juan D. Godino, Salvador Llinares y Vicenç Font.

\section{Referencias}

BREDA, A.; FONT, V.; PINO-FAN, L. R. Criterios valorativos y normativos en la Didáctica de las Matemáticas: el caso del constructo idoneidad didáctica. Bolema, Rio Claro, v. 32, n. 60, abr. 2018, p. 255-278.

CASTRO, W. et al. Mathematics Education research agenda in Latin America motivated by coronavirus pandemic. EURASIA Journal of Mathematics, Science and Technology Education (en prensa).

DIBNER, K. A.; SCHWEINGRUBER, H. A.; CHRISTAKIS, D. A. Reopening K-12 Schools during the COVID-19 Pandemic: A Report from the National Academies of Sciences, Engineering, and Medicine. JAMA - Journal of the American Medical Association, v. 324, n. 9, set. 2020, p. 833834.

JOSHI, D. R. Influence of ICT in mathematics teaching. International Journal For Innnovative Research in Multidisciplinary Field, v. 3, n. 1, jan. 2017, p. 7-11.

LABORDE, C. The role and uses of technologies in mathematics classrooms: Between challenge and modus vivendi. Canadian Journal of Science, Mathematics and Technology Education, v. 7, n. 1, 2007, p. 68-92.

MIARKA, R.; MALTEMPI, M. O que será da Educação Matemática depois do Coronavírus? Bolema, Rio Claro, v. 34, n. 67, ago. 2020, p. ii-iv.

PRATES, U.; MATOS, J. F. Mathematics Education and Distance Learning: a systematic literature review. Bolema, Rio Claro, v. 34, n. 67, ago. 2020, p. 522-543.

VAN LANCKER, W.; PAROLIN, Z. COVID-19, school closures, and child poverty: a social crisis in the making. Lancet Public Health, v. 5, n. 5, april 2020, e243-e244.

VERSCHAFFEL, L.; DEPAEPE, F.; MEVARECH, Z. Learning Mathematics in Metacognitively Oriented ICT-Based Learning Environments: A Systematic Review of the Literature. Education Research International, 2019.

ZHANG, W. et al. Suspending Classes Without Stopping Learning: China's Education Emergency Management Policy in the COVID-19 Outbreak. Journal of Risk and Financial Management, v. 13, n. 3,2020, p. 55 .

ZHOU, L. et al. F. School's Out, But Class' On', The Largest Online Education in the World Today: Taking China's Practical Exploration During The COVID-19 Epidemic Prevention and Control As an Example. Science Insights Education Frontiers, v. 5, n. 2, 2020, p. 501-516. 